\title{
A PREDICTIVE MODEL OF RIDE SATISFACTION IN MASS TRANSIT USERS
}

\author{
Joseph J.P. Simons, Ph.D. \\ Ilya Farber, Ph.D. \\ Social and Cognitive Computing Department, \\ Institute of High Performance Computing, Singapore
}

$13 / 08 / 2018$

Author note. Correspondence concerning this article should be addressed to Joseph J.P. Simons, Institute of High

Performance Computing, Agency for Science, Technology and Research, 1 Fusionopolis Way, \#16-16 Connexis North,

Singapore 138632. Contact: (65) 6419 1423, simonsj@ihpc.a-star.edu.sg. 
ABSTRACT. We describe a model for predicting subjective user satisfaction based on objective ride conditions. The model takes ride features (e.g., duration, crowding, waiting time) as inputs and estimates users' single-ride satisfaction. This model enables transit authorities to predict the effect of service changes on user satisfaction. This in turn makes it possible to compare multiple candidate system configurations to determine which would yield the highest level of satisfaction. Using a sample of mass rapid transit users $(n=641)$, the model is first trained then validated against unseen data. Prediction uncertainty is accounted for using a residual term. We also provide an example of how this model can be used to derive predictions, using artificial data.

\section{Highlights}

- We derive a predictive model of single-ride satisfaction from a diverse sample of transit users ( $n=641)$.

- Transit authorities can use this model to predict the impact of changes to service on rider satisfaction.

- Validation analysis is provided to confirm the model's applicability to new cases. 


\section{INTRODUCTION}

User satisfaction is a major consideration in operational decisions about transit networks. When considering changes to services, a central question is which option will cause the biggest increase (or smallest decrease) in satisfaction - especially when trade-offs across different aspects of service are unavoidable. For example, imagine adding more trains to a service reduces crowding but also decreases service consistency (due to train congestion at stations). Would this change be worth making? To inform decisions such as these, a model is needed to map changes in objective service levels onto user satisfaction.

Here we describe our process for building a predictive model of transit users' satisfaction. The model takes key features of a ride as input and generates an estimate of how satisfied a user would be with that ride. This model is empirically calibrated against a sample of mass rapid transit users $(n=641)$ and incorporates an empirically-derived residual term to capture the influence of non-modelled factors.

An additional benefit is the insight generated into ride-specific drivers of satisfaction. In calibrating the model, we need to assign weights to different ride features (e.g., average crowding level, minutes of waiting time). These weights indicate how important the features are to passengers. Therefore, the model can be used to assess which features are most consequential and quantify trade-offs between features. For example, we can ask how many additional minutes of travel time being seated is worth. These results are therefore both relevant for understanding passenger psychology and for targeting service improvements.

\subsection{SYSTEM CHANGES, RIDE EXPERIENCES, AND PASSENGER SATISFACTION}

The operational decisions of transit authorities have consequences for the objective ride experiences of passengers. When changes are made to services, the speed of travel, consistency of journey times, and level of crowding can all potentially change. Of course, these changes will rarely be straightforward. Due to the interdependent nature of transit networks, the effects are often complex and both spatially and temporally heterogenous. A given change may have surprising ripple effects affecting unforeseen locations, aspects of service, or time points. 
Predicting the effects of service changes on objective ride experiences is becoming more feasible via the use of agent-based models (ABMs). These computational models simulate each vehicle and/or passenger as an independent agent, and so capture aggregate trends by modeling individual-level behaviours and interactions (McDonnell \& Zellner, 2011; Othman, Legara, Selvam, \& Monterola, 2015; Wahba \& Shalaby, 2011). This micro-level simulation means ABMs are able to capture higher-level phenomena which emerge from lower-level interactions and feedback loops. For this reason, they are well suited to predicting how changes to service will propagate through an interdependent transit network.

However, what is operationally most important is forecasting effects on customer satisfaction. Satisfaction is an important metric for assessing service quality, and so often quantified alongside more objective performance indicators (Eboli \& Mazzulla, 2011; Stradling, Anable \& Carreno, 2007; Tyrinopoulos \& Aifadopulou, 2008). Therefore, in making operational decisions, it would be most useful if simulations were able to predict not only objective experiences but also the resulting satisfaction levels. Imagine operators had the option to add more operational trains, which a well-validated simulation suggests will decrease train crowding by $\sim 20 \%$ but increases the risk of delays by $\sim 10 \%$ (as trains are more likely to have to wait outside stations). The operational decision is whether to switch to the new schedule. In making this decision, it would be extremely informative to know whether the passengers would, on average, be more or less satisfied with the new service.

To make these predictions, a model is needed to connect objective ride experiences with passenger satisfaction. This function would take a passenger's ride experiences as an input, and output their satisfaction level. Such a model could be connected to predicted changes in objective experiences, whether derived from an ABM or other methods. The primary benefit is better operational decisions by forecasting how system changes affect passenger satisfaction. In addition to this predictive value, the weights that the model assigns to different factors would provide insight into the drivers of satisfaction. 


\section{Crowding level}
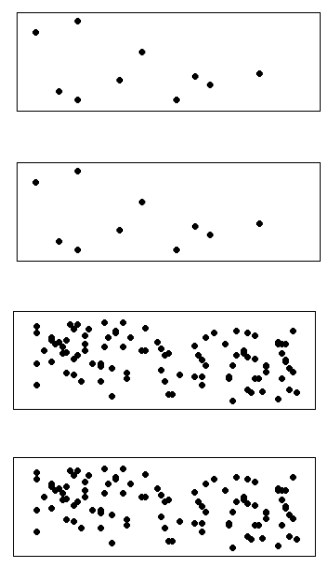

Ride
duration
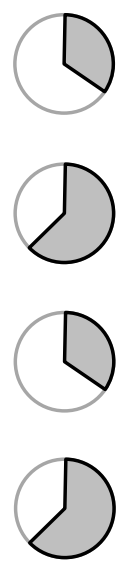

\section{Waiting \\ time}

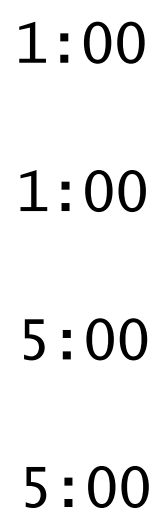

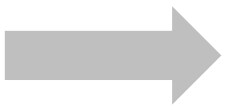
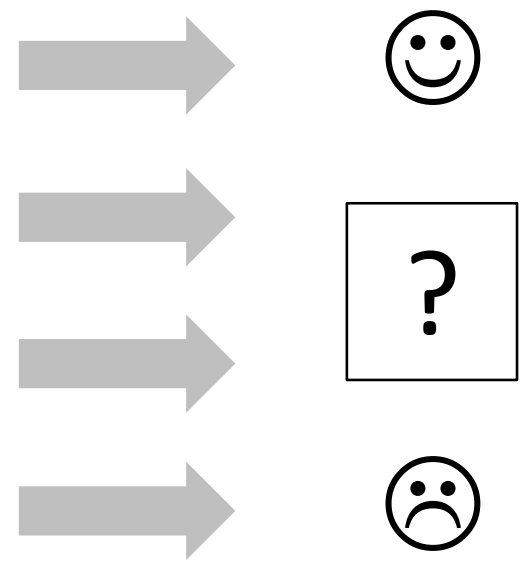

Figure 1. Goal of satisfaction model. The aim is to predict how satisfied users will be with particular combinations of ride conditions (in this example crowding, speed, and waiting time). Such a model would enable transit authorities to estimate how changing service will affect user satisfaction.

\subsection{PRIOR LITERAURE: IDENTIFICATION OF CAUSES OF SATISFACTION}

Prior work in this area has been directed towards the goal of identifying drivers of satisfaction. For this reason, the research designs are not well suited for building predictive models. Below, we outline four literatures which have addressed causes of satisfaction.

Subjective evaluations of service aspects. Which aspects of service are the strongest associates of overall satisfaction? To answer this question, researchers collect subjective evaluations of individual service aspects - such as rated satisfaction with speed, reliability, and so forth - and overall satisfaction. These can then be statistically related using path analyses or structural equation models. Such models have been used to study satisfaction in groups as diverse as subway-using residents of New York City (Stuart, Mednick, \& Bockman, 2000), bus-riding students in Cosenza, Italy (Eboli \& Mazzulla, 2007), and private paratransit users in Bandung, Indonesia (Joewono \& Kubota, 2007). 
The results from these models can be extremely useful for strategically prioritizing improvements. For example, using data from a routine customer satisfaction survey of bus users in Granada, de Oña, de Oña, Eboli and Mazzulla, (2013) found service factors were four times more important than comfort factors in explaining satisfaction.

While they have important uses, models based on subjective evaluations are not ideal for making specific predictions about the effects of service changes. Changes in objective conditions and satisfaction with aspects of services are unlikely to show a linear association. Imagine a hypothetical schedule would save users five minutes on their travel time. How much more satisfied with the system's speed would they be? Whatever the answer, it is probably not equal to five times the benefit of saving one minute. ${ }^{1}$

Ride vs. rider causes. Work in this area collects data on objective ride conditions (e.g., time spent, travel mode, waiting time) alongside individual characteristics of users (e.g., personality, attitudes, general well-being). Satisfaction is then modelled as a result of both sets of factors. Generally this is done using surveys (Ory \& Mokhtarian, 2005; St-Louis, Manaugh, van Lierop, \& El-Geneidy, 2014). However, recently Carrel and colleagues have used a similar approach to combine survey data (on personal characteristics and satisfaction; Carrel, Segupta, \& Walker, 2017) with smartphone location tracking (for objective ride conditions; Carrel, Lau, Mishalani, Sengupta \& Walker; 2015) to produce an integrated model (Carrel, Mishalani, Sengupta \& Walker, 2016)

These studies give important insight into drivers of satisfaction. They highlight factors beyond level of service which correlate with satisfaction (e.g., using the commute to do other tasks). Those which compare modes (Ory \& Mokhtarian, 2005; St-Louis, Manaugh, van Lierop, \& El-Geneidy, 2014) also reveal how the same factor can have different effects for different kinds of travel. For example, variability in travel time seems to be more of an issue for commuters who drive than for those who walk/cycle or take trains.

However, these studies are limited in their ability to make predictions for new cases. First, they often only consider a subset of ride factors. The papers above all consider travel time and some measure of service reliability (most directly quantified in Carrel et al., 2016a). However, none include a direct measure of crowding or seating availability on transit, which are surely

\footnotetext{
${ }^{1}$ This point is emphasized by the work of Friman and colleagues arguing satisfaction is related to the occurrence of particular negative events (Friman, Edvardsson \& Gärling, 2001; Friman, \& Gärling, 2001; reviewed in more detail below).
} 
important factors for any projective model to consider. Second, from the perspective of simulations, personal characteristics are really just another source of random variation. We can meaningfully simulate changing ride conditions, but personal characteristics would have to be randomly sampled from a representative population distribution. This would require largescale surveys of the population, while not adding any ability to detect differences between schedules.

Comparing across cities or time. A third literature takes a comparative approach to user satisfaction. Rather than looking at individual rides, aggregate user satisfaction is compared across cities (Friman \& Felleson, 2009; Friman, Fujii, Ettema, Gärling, \& Olsson, 2013) or before and after changes to service (Friman, 2004). Due to the use of natural variations in service quality and random sampling of respondents, these results have impressive levels of ecological validity.

Overall, these studies are able to detect differences in satisfaction, but have a difficult time explaining them. There are significant satisfaction differences between cities (Friman \& Felleson, 2009; Friman, Fujii, Ettema, Gärling, \& Olsson, 2013). However, these differences are not well explained by differences in service metrics (Friman, 2004). Furthermore, comparing within regions, quality improvements did not lead to an increase in satisfaction. Indeed, to the extent there were changes, these were negative - possibly due to an increase in negative events, although this was not directly tested (Friman, 2004).

For predictive purposes, these studies are limited by their narrow range of explanatory variables and by conflating transit systems with rides. First, they include fairly limited measures of system performance. Thus, they are often not able to predict the effect on satisfaction from changing a given aspect of service. Second, they measure satisfaction with the transit system as a whole. This construct is very broad; much broader than satisfaction with a given ride. First, there are factors (such as network coverage) which will determine satisfaction with a transit system but which cannot be attributed to any given ride. Second, there is the issue of ride aggregation. Even if we could establish how "good" or "bad" a series of rides are, it is unlikely these could be simply summed to provide an overall satisfaction score. For example, negative rides may carry more weight, there may be threshold-like effects, or more recent experiences may be more important.

Specific events. Finally, there is literature examining the effects of specific ride events. One particular area of focus is negative critical incidents - cases where service falls below expected standards. Friman, Edvardson, and Gärling (2001) show that recalled frequency of these incidents predicts overall system satisfaction. Friman and Gärling (2001) provide stronger 
causal evidence by using a stated-preference design (where respondents are presented with systematically-varied scenarios and rate their satisfaction with each). Ettema, Gärling, Eriksson, Friman, Olsson, and Fujii (2011) use a similar design varying effects of waiting and travel time (although their primary goal was to validate a measure of satisfaction rather than quantify the effects of these factors). Finally, Ettena, Friman, Gärling, Olsson and Fujii (2012) examined the effects of selfreported in-travel activities on satisfaction with the journey.

The purpose of these studies is to establish whether a set of factors influences satisfaction, rather than to build a comprehensive model of satisfaction. For this reason, the authors generally do not include a broad range of predictors. This limits their use for prediction. The study which comes closest is Friman, Edvardson, and Gärling (2001), who measured frequency of negative incidents across a range of service dimensions. This study is also notable for making a solid attempt at answering how experiences across multiple rides are integrated into a single satisfaction scores. However, even in this case, more continuous measures of service provision - factors such as average crowding level or travel speed - were not included. Thus, the model may be missing the predictive ability which these variables can provide. Furthermore, rather than measuring concrete rates of occurrence, the study relied on respondents rating frequency of negative incidents in terms of broad categories (never / a few times / occasionally / regularly).

\subsection{BUILDING A PREDICTIVE MODEL: RESEARCH DESIGN AND ANALYSIS}

Our goal is to build a model which can generate satisfaction predictions from simulations of different ride features. The central idea is that ABMs can provide rich information about the likely experiences of transit users, but this information needs to be translated into effects on satisfaction. This goal informed our research design and analysis. The data we gathered needed to be suitable to generate such a model, and the analysis needed to assess how well the model will perform on new cases.

\subsubsection{RESEARCH DESIGN CONSIDERATIONS}

Given the goal of prediction, our research design has three key features. While we don't claim originality for any of these in isolation, we are not aware of any other research which combines all three. 
1. We model satisfaction as resulting from concrete ride experiences rather than broad evaluative dimensions.

2. We consider a wide range of predictions.

3. We attempt to predict satisfaction with a particular ride, rather than with the system as a whole.

Concrete ride experiences. Our predictors are objective ride features, rather than subjective evaluations. We asked respondents about the specific details of their most recent ride, concentrating on quantifiable events and features. Thus, for example, we asked whether the train stopped between stations rather than an overall rating of reliability. The resulting model will be able to generate predictions based on actual on-the-ground experiences. In this way, we avoid having to make assumptions about how concrete experiences are translated into subjective evaluations of ride dimensions.

Broad range of predictors. Rather than focusing on a subset of ride features, we included a broad swathe of predictors. The resulting model will be able to take into account a wide range of factors. Generally speaking, the larger the range of factors included as predictor candidates, the greater the chance the model will be able to recreate the variability between riders. (Of course, a more complex model raises questions about generalizability, as discussed in the next section).

Satisfaction with rides. Finally, we asked respondents about their satisfaction with their most recent ride, rather than overall satisfaction with the system. Ride satisfaction is relevant to assessing system performance and is plausibly predictable from ride features. Predicting overall system satisfaction would be a much more complex task, requiring tracking multiple rides over an individual, finding an appropriate function to integrate them, as well as taking account of non-ride contributors to satisfaction (e.g., network coverage).

\subsubsection{ANALYSIS: ACCURACY AND GENERALIZABILITY}

Even a well-calibrated mode will not perform perfectly. This inaccuracy can be traced to at least three sources. First, the model coefficients are estimates based on sample data, and hence may deviate somewhat from the true population values. In particular, they may be too tightly fitted to the sample data, and so capitalize on chance relationships in that sample (so-called "overfitting"; more complex models have greater potential to over-fit). Overfitting is particularly worrisome as it means the model may perform well on the current dataset but much less well on new cases. Second, a psychological outcome such as 
satisfaction will only be measured with a certain degree of accuracy. Less than perfect measurement will introduce random noise into the model, which attenuates the accuracy of the model. (Imagine trying to answer a physics problem when all the measurements have random perturbation added to them). Third, any model will only include a subset of relevant causal factors. Satisfaction will be driven by far more factors than can be practically included in a model, and the remaining unmodelled drivers will show up as prediction error.

Model inaccuracy raises a number of issues. First, an appropriate model needs to be selected. If there is evidence of overfitting, a more regularized model can be used. Such a model would work less well on the training data, but would generalize better to new cases. Second, the generalizability of the final model needs to be assessed. Third, there is the question of how the resulting uncertainty in predictions should be best represented. If the model is to be used for operational purposes, the associated uncertainty needs to be accurately assessed and communicated to the model's users.

To these ends, we took three steps.

1. We use cross-validation to select a modelling algorithm which shows low evidence of overfitting.

2. We utilize a separate test sample to assess the performance of the final model on new cases.

3. We add a random residual term to the final model, so that predicted values have an appropriate degree of variability.

Cross validation and model selection. We compared the performance of two modelling algorithms through a crossvalidation process. The two algorithms are standard multiple regression and lasso regression - a variant regression technique which penalizes complex models and hence is less susceptible to capitalizing on sample-specific relationships. Multiple regression gives a fuller model, whereas lasso regression gives simpler and more generalizable models. To compare these two, the data were repeatedly split, so that models could be fitted to a subset and then tested against the remainder. Repeating this process a number of times allows a comparison of how well each technique generalizes in the current case.

Test set and generalizability. Prior to the cross-validation process, a separate random subset of the data was set aside. This was not used at any stage of model training. Hence, once a modelling technique was chosen and a final model trained (using the whole training set), it could be tested on this entirely unseen data. This test provides an assessment of how well the model will perform on new data. 
Residual term. Finally, the variance of the prediction errors in the test set was used to add a random term to the final model. This term adds additional random noise to each prediction, providing a realistic degree of variability in the predicted scores. Thus, when the model is used to simulate different scenarios over a large number of users, the resulting scores will have an appropriate range, capturing the degree of uncertainty in the predictions. 


\section{MATERIALS AND METHODS}

\subsection{QUESTIONNAIRE INSTRUMENT}

The predictive model was based on data from a larger-scale satisfaction study of Singapore's Mass Rapid Transit (MRT) system. The MRT is a city-spanning metro rail network, which averaged over 3.9 million rides per day in 2016 (Singapore Land Transit Authority, 2017). In the relevant section of the survey, respondents rated their most recent ride. First, they indicated a number of features of this ride (e.g., wait time, crowding level). Subsequently, they rated their satisfaction with the ride using two items.

Ride \& rider features. The items used to measure features of the ride and respondent are given in Table 1 . In the current analysis, we focus on features which are likely to be affected by system changes as well being amenable to forecasting. The full questionnaire measured a wider range of ride features, many of which may be relevant to satisfaction. However, we did not include those which were unlikely to be affected by system changes and which would be difficult to include in a simulation. An example is thermal comfort - while the temperature is likely relevant to satisfaction, decisions such as scheduling changes are unlikely to affect the temperature and it is unlikely to be included in simulations of service changes.

Satisfaction. Transit satisfaction was measured using two items given in Table 2. One relates to cognitive evaluation, whereas the other is affective. Thus, while shorter than the satisfaction with transit scale (Ettema et al. 2011; Friman et al., 2013), the current measure does cover the same domains (i.e., thoughts and feelings). To minimize the effects of acquiescence, one item was positively valenced and the other was negatively valenced. The negative item was reversedcoded.

Versions. To maximize participation, both paper and online versions were created. The paper versions were to be completed in person, and were available in both English and Mandarin Chinese. The online version had some minor changes to question formats as necessitated by the medium, and was also only available in English. The online version was created using the SurveyGizmo website and was mobile-responsive (i.e. would be appropriately formatted regardless of whether respondents used a computer, tablet, or smartphone to access it). 
Table 1

Items assessing features of most recent ride. Left hand column gives feature name, right hand column gives question wording (in bold), response options, and how scores were converted

\section{TIMING}

Timing - AM peak

Timing - PM peak

Timing - weekend
"On what day and time did you tap in for this ride?"

Checkboxes for days, free response for time

Dummy coded using the following categories

Weekday off peak (reference category); Weekday AM peak (7.30am-9.30am);

Weekday PM peak (6.00pm-8.00pm); Weekend (any time)

\section{DURATION}

Total

Longer than expected

Shorter than expected
"Approximately how long did your most recent ride take from tap in to tap out?

Checkboxes for $<10 / 10-20 / 20-30 / \ldots / 80-90 / 90+$ mins

Coded by midpoint of categories ( 5 mins for first, 95 for highest); divided by 10 to use 10-min units

"Overall, did your most recent ride take"

Checkboxes for Less / About the amount / More time than you expected

Dummy coded with middle category as reference category

\section{WAITING TIME AND TRANSFERS}

Waiting time

Transfers
"On your most recent ride, how long did you have to wait for a train to arrive? Please indicate how long you waited at the first station, and during any subsequent transfers."

Check boxes $<2$ mins / 2-5 mins / 5-10 mins / $10-15$ mins / > 15 mins / Not sure

Separate responses for first station and any subsequent transfers

Waiting time. Coded by midpoint of response category for first station ( $1 \mathrm{~min}$ for first, 17.5 for > 15)

Transfers. Coded by number of transfers times given

\section{CROWDING \\ Average \\ Worst}

"On average, how crowded was the train during your most recent ride? "

"At its worst (i.e., most crowded point), how crowded was the train during your most recent ride?"

Both had check boxes with the following options:

Some seats were available

All seats were occupied and some passengers had to stand

Crowded but some open space in the inner part of the carriage

Crowded throughout the carriage

Very crowded throughout the carriage, some passengers pressed against the doors

Responses converted into density estimates based on Lam, Cheung, \& Lam, C. F. (1999).

\section{POSITIONING}

Seated

Middle of carriage

\section{UNABLE TO BOARD}

Occurrence

Number

"For most of the time in your most recent ride, where were you located?"

Checkboxes for seated / standing close to the doors / standing in the middle of the carriage away from the doors Dummy coded with middle category as reference category

"During your most recent ride, were there any trains that were so crowded you were unable to board? If so, how many?"

Yes / No response, with free response if they said yes

Occurrence - 1 if "yes", 0 if "no"

Number - 0 if "no", number listed if "yes"

\section{UNSCHEDULED STOPS}

Occurence

"Did the train have to stop between stations during your most recent ride?"

Duration

Yes / No response with free response if they said "yes"

Occurrence - 1 if "yes", 0 if "no"

Number - 0 if "no", number listed if "yes". Values over 10 coded as 10.

\section{DEMOGRAPHICS \\ Senior}

"How old are you?"

Check boxes for 5-year ranges from 15-19 to 75-79, plus an $80+$ box

1 if 60 years or above, 0 otherwise

Student

Checkboxes for various options

1 if "Education (full-time or part-time)", 0 otherwise 
Table 2

Items assessing satisfaction with most recent ride

$\begin{array}{ll}\text { SATISFACTION } & \text { "Overall, how good was your most recent ride?" } \\ \text { Cognitive } & \text { Check boxes } 1 \text { (Very bad) - } 7 \text { (Very good) } \\ \text { Affective } & \text { "How unhappy were you with your most recent ride?" } \\ \text { Check boxes } 1 \text { (Very happy) - } 7 \text { (Very unhappy) }\end{array}$

\subsection{DATA COLLECTION}

Data collection was conducted between 14 September and 13 November 2015. Recruitment was performed outside MRT stations by undergraduate assistants recruited from local universities. During each data collection session, potential respondents were either approached to complete the paper version at the station (in-person sessions) or given flyers with a link to the online versions (online sessions). Recruitment types were mixed within sessions, but in distinct time blocks - i.e., a given respondent was not presented with a choice between the in-person or online versions. In terms of inducements, inperson participants were offered a voucher for a local coffee shop, whereas online participants were entered into a draw for a larger-denomination voucher for a local supermarket. This difference was due to the difficulty of providing individual rewards to online participants. 


\section{RESULTS}

We report our results in three sections. First, we provide a full regression model, estimating the effects of all ride features. In addition to its potential predictive value, this comprehensive model gives insights into the drivers of satisfaction and so is discussed in some detail. Second, turning back to the issue of prediction, we report a comparison of different modelling techniques (multiple and lasso regression) using cross-validation. We also describe the performance of our final model on unseen test data. Finally, to demonstrate the utility of the model, we show how it can be used to derive predicted satisfaction values under two competing schedules.

The analysis was conducted using R version 3.1.3 (R Core Team, 2015). Dividing the data, cross-validation, and coordinating model training was performed using the caret package (v6.0.68; Kuhn, 2008). Lasso regression was estimated using the glmnet package (v 2.0.5; Friedman, Hastie, Tibshirani, 2010) .

\subsection{SAMPLE CHARACTERISTICS AND DATA PREPARATION}

Demographics. A summary of demographics is given in Figure 2. Comparative figures from the 2010 Singapore census are also shown. As can be seen, our samples were broadly representative, although with some over-representation of younger respondents.

Avoiding outliers. For three features, steps were taken to avoid very high values (which would be overly influential in subsequent analyses). For total ride duration and waiting time, the open-ended upmost categories were coded by assuming using the same width as the prior category. For example, a " $90+$ mins" was coded as 95 minutes, as the prior response option was 10 minutes wide (80-90 minutes). In the case of the duration of unscheduled stops, the four responses over 10 minutes were coded as 10 minutes.

Summary statistics. Table 3 gives the means, standard deviations, and inter-correlations for all variables included in the analysis. Note these are prior to any imputation of missing data or the split into training and test sets (described below). 
Missing data. The 74 records missing satisfaction scores were dropped from the analysis. This was to ensure satisfaction scores remain a ground-truth variable to test against - if a person has a 5 for satisfaction, we know that is their genuine response and not an imputed value. However, when a record was missing a value on a ride feature, we substituted the median value for that feature. Using an average value is a fairly conservative strategy, and the median was used as many features should only take integer values. Under this procedure, a sub-optimal imputation process might harm model performance (as a different imputation procedure might give better estimates of the missing values) but it will not spuriously increase model fit (because the outcome variable is not imputed). The cases missing a satisfaction value were dropped prior to the split into training and test sets, and imputation was performed after this division. Hence, separate medians were calculated for the two sets.

Training and test sets. The target was a 70/30\% split training and test sets with stratification on ride satisfaction. After dropping cases missing satisfaction, there were 641 total cases. With stratification on satisfaction, these were split into a training set of 449 and a test set of 192.

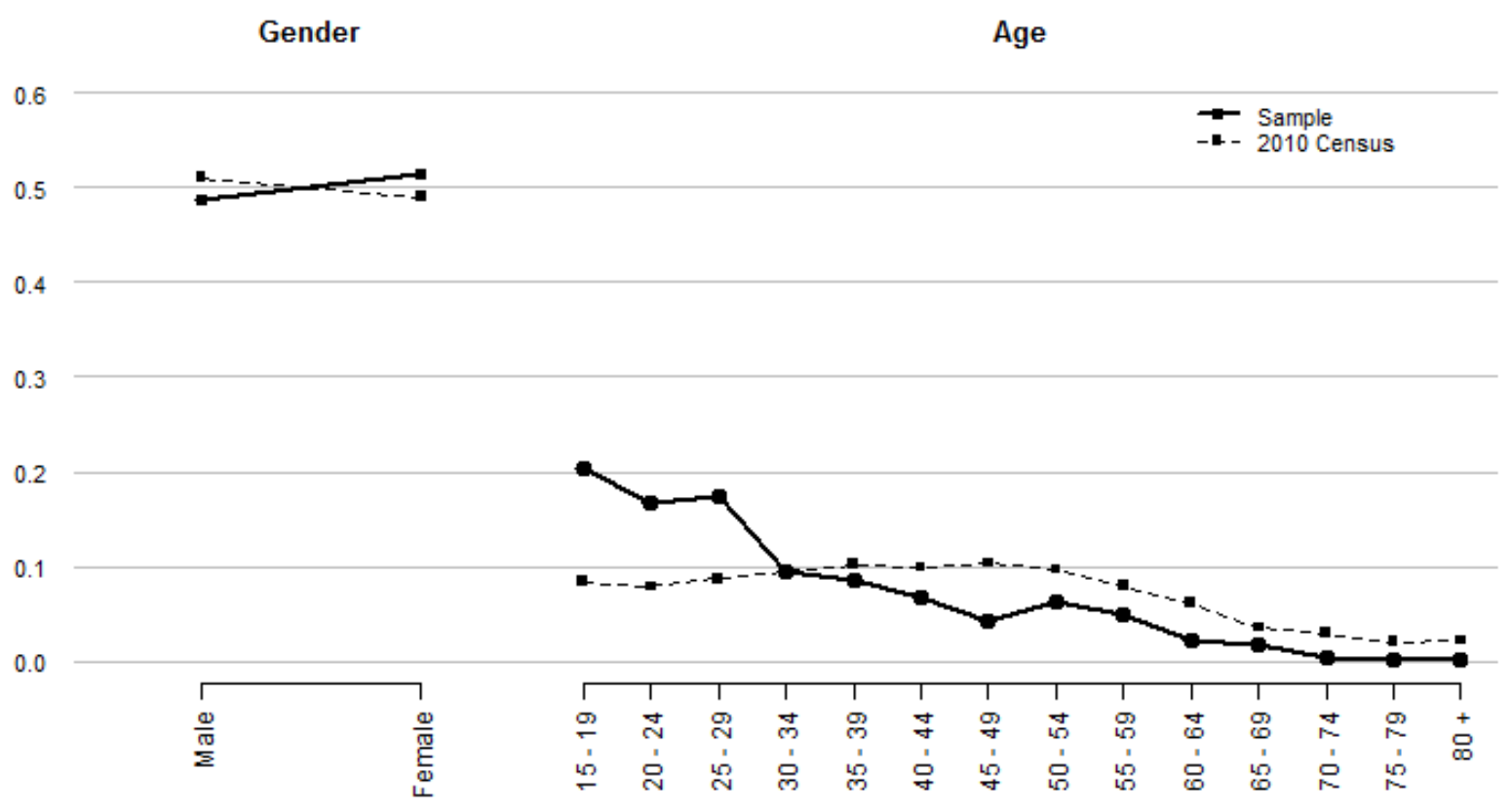

Figure 2. Demographics of sample. Solid lines show the proportion of the sample falling in each gender / age category. Dotted lines show representative figures from the census for comparative purposes. 


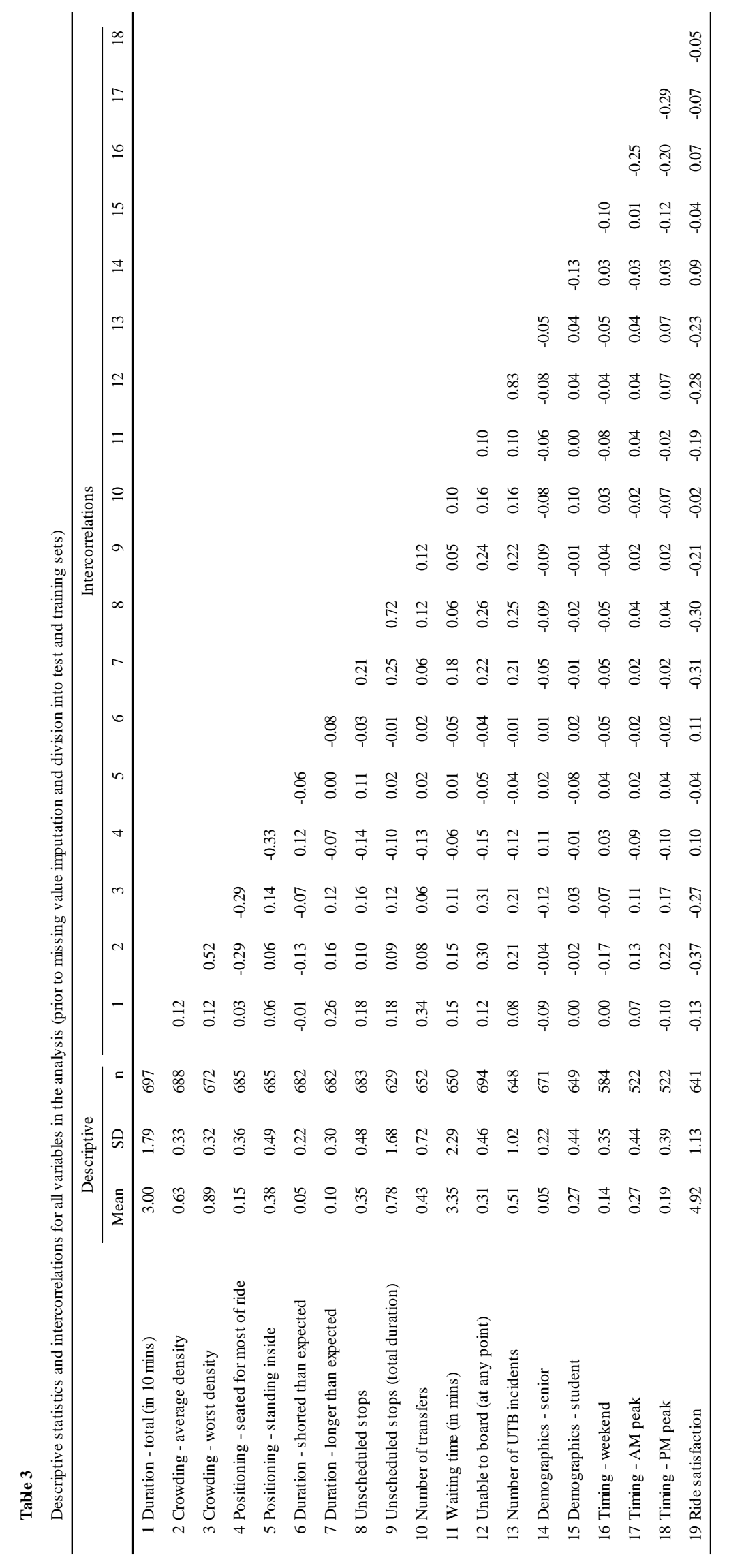




\subsection{INSIGHTS INTO SATISFACTION}

To determine how strongly each ride feature was associated with satisfaction, a multiple regression was conducted on the training data. The results are given in Table 4 . For each feature, we provide both the unstandardized and standardized regression coefficients.

The unstandardized coefficients come from performing a regression on the raw data. Each coefficient indicates the change in satisfaction associated with a one-point change in the ride feature. Hence, these coefficients are useful for anticipating the effect associated with a change in service quality. However, as they incorporate information about variance, these coefficients across variables does not provide a good guide to feature importance. For example, if we expressed ride duration in minutes (rather than 10 minute units), it would still make the same contribution to the model. However, the coefficient would become 10 times smaller.

The standardized coefficients are derived by first transforming all variables to have a mean of 0 and a standard deviation of 1 . In this model, the intercept term will be 0 and hence is omitted. The initial transformation puts all variables on the same scale. As a result, these coefficients are less informative for each individual effect but can be directly compared across features. A feature with a coefficient of 0.60 is twice as important as one with a coefficient of 0.30 .

In terms of inferential statistics, we also provide a 95\% confidence interval for each standardized coefficient and p-values. The conventional threshold for significance is a p-value of 0.05 , but the number of predictors necessitates a correction to control the error rate. Hence, we indicate whether each effect is significant after applying a Bonferroni correction.

As can be seen, three effects emerged as notably more important than the others: average crowding level; stopping between stations; and taking longer than expected. These effects had larger standardized coefficients than the others, and were the only three to emerge as statistically significant (when correcting for multiple comparisons). Thus, we can say that people are substantially less satisfied with rides which are crowded, involve unexpected stops between stations, and which take longer than expected. Furthermore, when targeting service improvements, improving these outcomes would lead to the biggest increases in user satisfaction. 
There was one surprising finding. Being seated was associated with a decrease in satisfaction, an effect which reached the .05 level of significance. While this effect may be genuine (possibly due to the social dilemma of whether to give the seat to needy others), there are two reasons to think it is an artefact. First, it only holds controlling for other factors. If no other predictors are taken into account, being seated has a positive association with satisfaction (albeit not reaching significance; $b$ $=0.28, \mathrm{p}=.07)$. Second, it does not survive the Bonferroni correction for multiple comparisons. Hence, we do not have strong evidence for concluding there is an effect in the wider population. These two points are consistent with the negative coefficient being a result of sample-specific noise, especially in the correlations between predictors.

Table 4

Regression coefficients for all features

\begin{tabular}{|c|c|c|c|c|c|c|}
\hline \multirow[b]{2}{*}{ Predictor } & \multirow[b]{2}{*}{ Unstand. } & \multirow[b]{2}{*}{ Stand. } & \multicolumn{2}{|c|}{$95 \% \mathrm{CI}$} & \multirow[b]{2}{*}{$\mathrm{p}$} & \multirow[b]{2}{*}{ Bon. } \\
\hline & & & Low & High & & \\
\hline (Intercept) & 6.37 & - & - & - & - & \\
\hline Crowding - average density & -0.94 & -0.26 & -0.36 & -0.17 & $<.001$ & $*$ \\
\hline Unscheduled stops (at any point) & -0.50 & -0.21 & -0.31 & -0.10 & $<.001$ & $*$ \\
\hline Duration - longer than expected & -0.70 & -0.17 & -0.25 & -0.08 & $<.001$ & $*$ \\
\hline Positioning - seated for most of ride & -0.34 & -0.10 & -0.19 & -0.01 & 0.03 & \\
\hline Unable to board (at any point) & -0.25 & -0.10 & -0.21 & 0.02 & 0.09 & \\
\hline Duration - total (per 10 mins) & -0.06 & -0.08 & -0.17 & 0.00 & 0.06 & \\
\hline Crowding - worst density & -0.29 & -0.08 & -0.18 & 0.01 & 0.09 & \\
\hline Waiting time (in mins) & -0.04 & -0.08 & -0.16 & 0.00 & 0.06 & \\
\hline Number of transfers & 0.13 & 0.08 & -0.01 & 0.16 & 0.08 & \\
\hline Duration - shorted than expected & 0.25 & 0.05 & -0.03 & 0.13 & 0.20 & \\
\hline Unscheduled stops (total duration) & 0.03 & 0.05 & -0.06 & 0.15 & 0.37 & \\
\hline Number of UTB incidents & -0.05 & -0.04 & -0.15 & 0.06 & 0.43 & \\
\hline Demographics - student & -0.11 & -0.04 & -0.12 & 0.04 & 0.32 & \\
\hline Positioning - standing inside & -0.09 & -0.04 & -0.12 & 0.05 & 0.39 & \\
\hline Demographics - senior & 0.20 & 0.03 & -0.05 & 0.12 & 0.41 & \\
\hline Timing - PM peak & 0.09 & 0.03 & -0.06 & 0.12 & 0.51 & \\
\hline Timing - weekend & -0.04 & -0.01 & -0.09 & 0.07 & 0.79 & \\
\hline Timing - AM peak & 0.01 & 0.00 & -0.08 & 0.09 & 0.96 & \\
\hline
\end{tabular}

N.B. Unstand. = unstandardized regression coefficient. Stand. = standardized coefficient. Bon. = whether effect remains significant when applying a Bonferoni correction for multiple comparisons. CI and p-values are for standardized coefficients. Standardized model omits intercept (as it will be approximately zero). 


\subsection{CREATING A PREDICTIVE MODEL}

The multiple regression model above is comprehensive in terms of ride features, but lacks information needed for predictive use. First, while it gives us the coefficients which best recreate the training data, we do not have an estimate of how well it would work on new samples. Second, the full model contains a large number of fairly small coefficients which cannot be statistically distinguished from zero (as is clear in Table 3). Knowing that these predictors have a negligible effect is useful for understanding the drivers of satisfaction. However, for predictive purposes, it would be extremely useful to know whether they can be dropped, and if so what the coefficients for the remaining effects should be. At the least, this would make the simulation, computational, and interpretive tasks easier, as there are fewer terms to deal with. However, a systematic process of feature selection can lead to a more generalizable model. These small effects may be capitalizing on sample-specific relationships, and hence in aggregate may pull off predictions - either directly or by distorting the coefficients of the larger effects.

To address these concerns, we compared the performance of a multiple regression model to lasso regression. Lasso regression attempts to balance accuracy with simplicity, and hence produces more parsimonious models. Complexity is defined as the absolute size of the coefficients. Hence, this algorithm seeks to both minimize error and make the coefficients as small as possible. As a result, it will often force trivial effects to zero, as the simplicity gains outweigh the negligible benefits to accuracy.

To facilitate generalizability, we present the results in terms of standardized coefficients. Unstandardized coefficients would give equivalent results for the current data. However, applying these to other transit systems would likely lead to misleading results, due to differently-distributed ride features. Imagine, for example, a transit system with much lower variability in crowding levels. In this case, a given change in crowding represents a more consequential shift - what might put a ride in the top $30 \%$ in our data may be in the top $10 \%$ in this other system. Hence, a given change in crowding should have a larger effect on satisfaction in this case. Standardized coefficients allow for these differences by expressing the effects of ride features in terms of standard deviation units. As we are using standardized units, the intercept term was omitted from all models. 


\subsubsection{CROSS VALIDATION}

To compare the generalizability of the two approaches, we used a repeated cross-validation procedure. For each algorithm, the training data was split into 10 segments. Each segment was then used to test a model trained on the remaining $90 \%$ of the data. This produces 10 values, each indicating how well a model estimated from $90 \%$ of the data generalized to the remaining 10\%. This process was repeated 10 times, splitting the data differently each time, and so giving 100 total results.

A further complication is that lasso regression requires a value for the trade-off between error and complexity $(\lambda){ }^{2}$ The most appropriate value to use will vary from dataset to dataset, so a process of tuning was used to identify good candidates. At every cross-validation fold, models using different values of $\lambda$ were tested, and their performance recorded. Thus, for multiple regression we get 100 performance scores, whereas for lasso regression we get 100 performance scores for every value of $\lambda$ tested. We use these scores to identify two values of $\lambda$. The "Best" score is the one which gives the best performance on average. We would use this value if we simply wanted to produce a model which would perform as well as possible on new data. However, we also identified the "One SE" score. This is the highest value which is within one standard error of best performance. The rationale here is that using this value will produce models which are simpler and whose accuracy is statistically indistinguishable from optimum. Hence, models produced using this value will be simpler and only negligibly worse in terms of accuracy.

Figure 3 shows the results of the tuning process. The $x$-axis shows tested lambda values, and the y-axis the average crossvalidated error. The black line shows the relationship between lambda and cross-validated error. The horizontal line gives the average cross-validated error for the multiple regression models as a reference value, and the vertical lines show the identified Best and One SE values.

\footnotetext{
${ }^{2}$ When $\lambda=0$, the algorithm only assess accuracy, and so the results are conceptually the same as a standard regression. When $\lambda=1$, the algorithm only assess simplicity, and hence will return zeroes for all coefficients. When $\lambda=0.5$, the two considerations are equally weighted.
} 


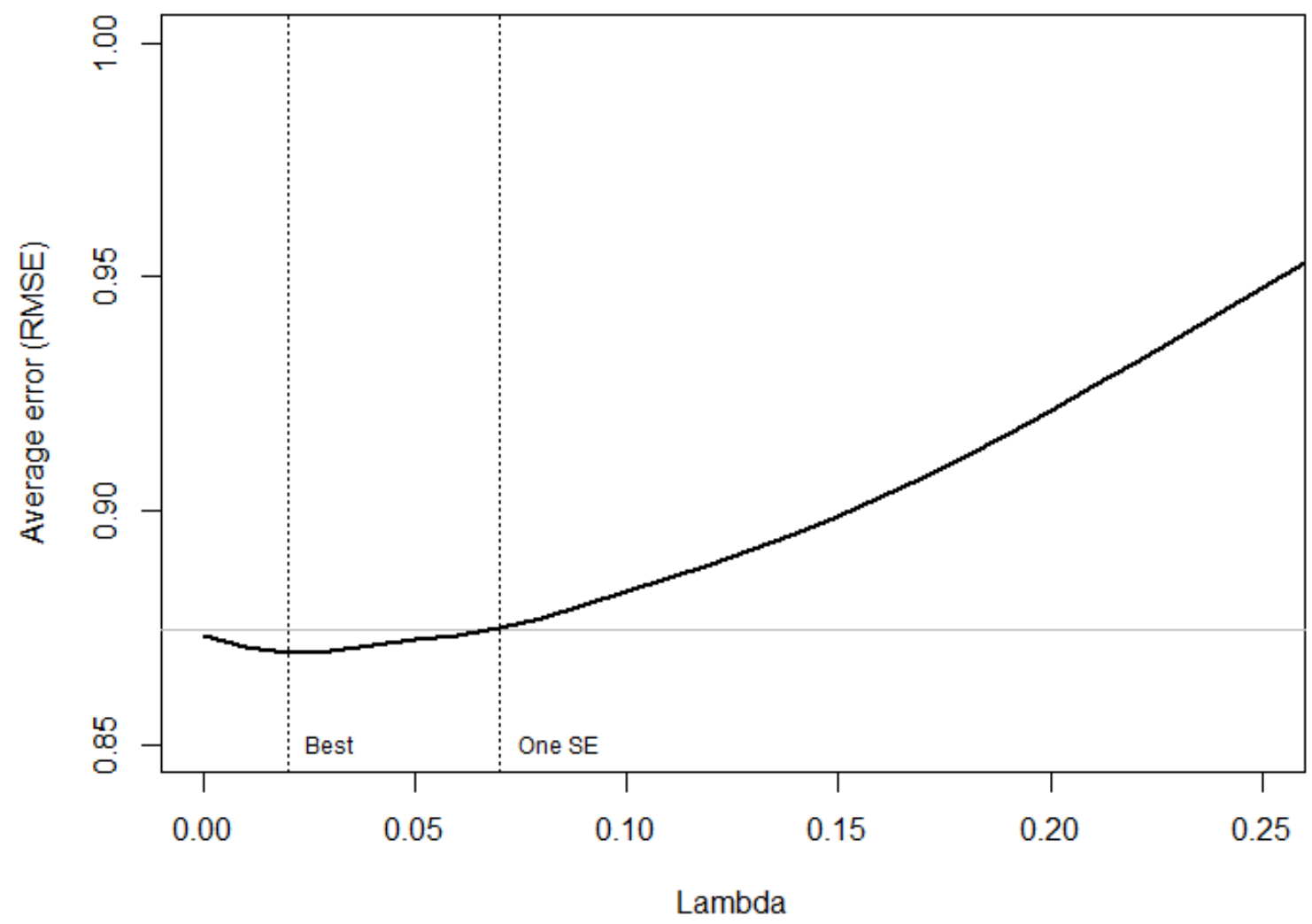

Figure 3. Results of tuning. The $x$-axis shows the tested $\lambda$ values and the $y$-axis the mean cross-validated error. The solid black line shows the mean cross-validated error for each tested value. The horizontal line shows the average value for multiple regression models, and the solid vertical lines the selected $\lambda$ values. Note the $\mathrm{x}$-axis has been truncated as the performance gets worse for the remaining values.

\subsubsection{CANDIDATE MODELS}

Table 5 summarizes the cross-validation results (in the top section) and shows the final model coefficients. The top section gives the average performance of multiple regression, lasso using the Best $\lambda$, and lasso using the One SE $\lambda$ across the cross validation runs. In the case of the RMSE, this is a subset of the information shown graphically in Fig 1 . The bottom section gives the coefficients when applying the three algorithms to the full training dataset. 
Table 5

Cross validation results and final models for multiple and lasso regression

\begin{tabular}{|c|c|c|c|}
\hline & \multirow[b]{2}{*}{ Multiple } & \multicolumn{2}{|c|}{ Lasso } \\
\hline & & Best & One SE \\
\hline \multicolumn{4}{|c|}{ Cross-validated fit } \\
\hline RMSE & 0.87 & 0.87 & 0.87 \\
\hline$R^{2}$ & 0.25 & 0.25 & 0.25 \\
\hline \multicolumn{4}{|c|}{ Model coefficients } \\
\hline Demographics - senior & 0.03 & 0.02 & . \\
\hline Demographics - student & -0.04 & -0.02 & . \\
\hline Timing - weekend & -0.01 & . & . \\
\hline Timing - AM peak & 0.00 & . & . \\
\hline Timing - PM peak & 0.03 & 0.01 & . \\
\hline Duration - total (per 10 mins) & -0.08 & -0.07 & -0.02 \\
\hline Crowding - average density & -0.26 & -0.24 & -0.21 \\
\hline Crowding - worst density & -0.08 & -0.07 & -0.04 \\
\hline Positioning - seated for most of ride & -0.10 & -0.06 & . \\
\hline Positioning - standing inside & -0.04 & -0.01 & . \\
\hline Duration - shorted than expected & 0.05 & 0.04 & . \\
\hline Duration - longer than expected & -0.17 & -0.16 & -0.15 \\
\hline Unscheduled stops & -0.21 & -0.17 & -0.14 \\
\hline Unscheduled stops (total duration) & 0.05 & . & . \\
\hline Number of transfers & 0.08 & 0.05 & . \\
\hline Waiting time (in mins) & -0.08 & -0.07 & -0.04 \\
\hline Unable to board (at any point) & -0.10 & -0.09 & -0.08 \\
\hline Number of UTB incidents & -0.04 & -0.03 & . \\
\hline
\end{tabular}

The most important point to note is that while the lasso models use fewer predictors, their cross-validation performance is broadly similar to multiple regression. This can be taken two ways. On the one hand, it implies it would be perfectly fine to use the full multiple regression model for predictive purposes. The fact multiple regression performs as well as lasso regression in cross-validation is evidence that it is not over-capitalizing on vagaries in the data. On the other hand, it also means that the more parsimonious lasso models can be used without harming predictive ability. The simpler models produced by these algorithms work as well on new data as the more comprehensive models generated by multiple regression. 
Moving forward, we use the One SE lasso model as our final model. As discussed above, all three estimation methods seem to work equally well. We therefore chose the simplest model, as that is the easiest to grasp conceptually and to implement in practice.

\subsubsection{VALIDATION AND MODEL FIT}

To provide a final estimate of how well the final model will work on new data, the One SE model was used to make prediction in the unseen test data. Fig 4a shows the results. As can be seen, there was a correlation between model-predicted and actual satisfaction values. The error value (0.89) was broadly consistent with the cross-validation results. These results provide evidence that the model will generalize to new data.
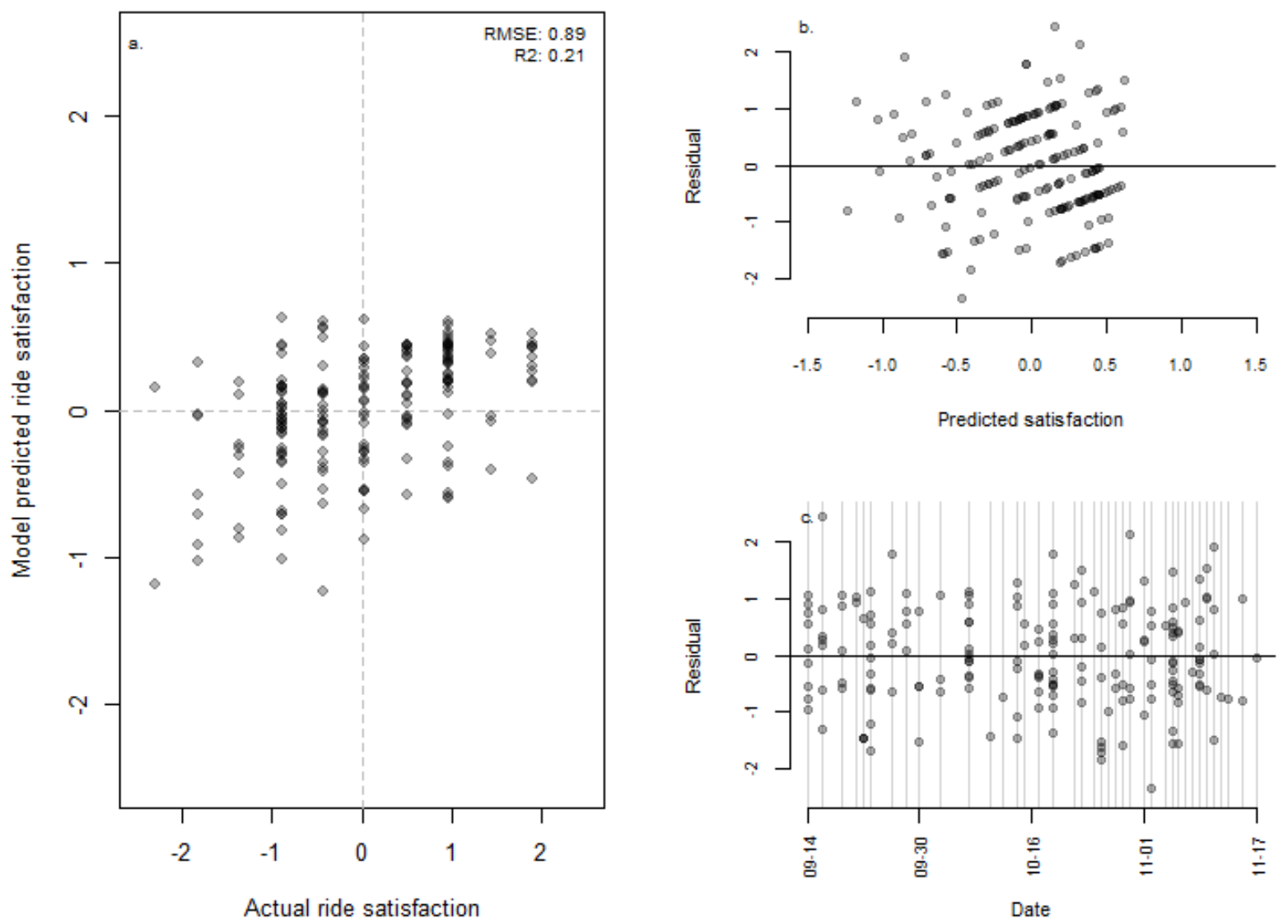

Figure 4. Validation on test data (a) and independence of residuals (b+c). (a) Accuracy of model on unseen test data. The $\mathrm{x}$-axis gives the actual observed satisfaction, and the $\mathrm{x}$-axis gives the values predicted by the model. Both values are deviations from the mean in standard deviation units. (b) Null relationship between model-predicted satisfaction (x-axis) and residual / model prediction error (y-axis). Note the x-axis was truncated at either end for ease of presentation. (c) Null relationship between date of data collection (x-axis) and residual (y-axis). 


\subsubsection{RESIDUAL TERM FOR PREDICTION}

To account for factors not included in the model, we include a term based on the prediction errors in the test sample. The rationale is as follows. If the model accounted for all factors which determined self-reported satisfaction, the model-predicted and actual satisfaction scores would be identical. Deviations from perfect prediction indicate the influence of additional factors not included in the model. These potentially include individual differences, additional aspects of ride experience (e.g., thermal comfort), and measurement error. In any given case, the residual represents the influence of all such external factors. The variability of these residuals therefore provides an empirical estimate of how much additional variability model-external factors are adding.

The RMSE from the test data was used to estimate the standard deviation of errors for new cases. Based on this estimate, an additional random term can be added to the final model, to represent the influence of model-external factors. This random term was modelled as normally distributed with a mean of 0.00 and a standard deviation of 0.89 . Including it captures the unpredictable effects of additional, non-modelled factors, and so gives a more representative distribution of scores at the aggregate level.

Notably, this approach assumes residuals are independent. The error for any one case is uninformative about the error for any other. We examined two potential sources of dependence. First, is the model systematically more likely to over- or underpredict satisfaction when it predicts high / low / medium scores? Second, are data points collected close together in time more likely to show errors in the same direction? If events such as service disruptions or weather are a major driver of satisfaction, we would expect data collected on the same day to show similar errors.

We examined both these possibilities by plotting residuals against the variables of interest, as shown in Figures $3 \mathrm{~b}$ and $3 \mathrm{c}$. Visual inspection suggests no relationships in either case. To test more formally, we fitted one-way ANOVAs using predicted score or date as an IV and residuals as a DV. In neither case was the $\mathrm{F}$ ratio significant: for predicted value, $F(156,29)=$ $1.06, p=0.44$; for date $F(38,146)=1.11, p=0.33$. As predicted value is a continuous measure, we also fitted a quadratic regression. Neither linear nor quadratic terms were significant, $B \mathrm{~s}=-0.21,-0.24, p \mathrm{~s}=.26, .48$. Thus, we did not find evidence for either form of dependence, and so proceeded to treat the residuals as independent. 


\subsection{USING THE MODEL: AN EXAMPLE OF PREDICTION}

One of the goals of the current model is to facilitate decisions between schedules which trade off different aspects of service.

To demonstrate this function, we compare two hypothetical schedules. The first represents baseline service, and one representing plausible effects of increasing the number of trains in service - lowered crowding, but increased unreliability (due to congestion at stations). However, the key point is to show how the simulated ride experiences can be turned into a satisfaction estimate, not to build an accurate simulation of how adding more trains would affect ride experiences.

For each schedule, we created a sample of 1000 transit rides. For each ride feature a probability distribution was specified, and values for each user drawn randomly from these distributions. The difference between the schedules was modelled by varying the means of the probability distributions, as shown in Table 6 below. The continuous predictors (duration, crowding, and wait time) were modelled as normally distributed, and capped at the maximum and minimum observed in our data. The dichotomous predictors (taking longer than expected, stops between stations, and being unable to board) were simply assigned a given probability of occurring on each ride. In actual use a more complex forecasting method would be necessary. However, for illustrative purposes this crude method is sufficient.

\section{Table 6}

Distribution statistics for generating ride features in simulated original and revised schedules

\begin{tabular}{|c|c|c|c|c|c|c|}
\hline & \multicolumn{2}{|c|}{ Mean } & \multirow[b]{2}{*}{ Diff } & \multirow[b]{2}{*}{ SD } & \multirow[b]{2}{*}{ Min } & \multirow[b]{2}{*}{$\operatorname{Max}$} \\
\hline & Original & Revised & & & & \\
\hline Duration - total (in 10 min units) & 3.0 & 3.0 & - & 0.50 & 0.50 & 9.50 \\
\hline Waiting time (in mins) & 3.5 & 3.5 & - & 1.00 & 1.00 & 17.50 \\
\hline Crowding - average density & 0.6 & 0.4 & -0.2 & 0.05 & 0.25 & 1.20 \\
\hline Crowding - worst density & 0.9 & 0.7 & -0.2 & 0.05 & 0.25 & 1.20 \\
\hline Duration - longer than expected & 0.1 & 0.2 & 0.1 & & & \\
\hline Unscheduled stops & 0.4 & 0.5 & 0.1 & & & \\
\hline Unable to board (at any point) & 0.3 & 0.3 & - & & & \\
\hline
\end{tabular}

NB The first four ride features (duration; waiting time; average and worst crowding) were treated as continuous and normally distributed with the mean, SD, and max/min values shown in the table. The final three (taking longer than expected; unscheduled stops; being unable to board) were treated as discrete events occurring with the probability shown in the "mean" column. Diff = difference in mean between original and revised schedules. 
As can be seen in Table 6, the revised schedule reduced crowding but increased unreliability. More specifically, the means of the two crowding variables were reduced by $20 \%$ of train capacity. However, the probability of taking longer than expected and unexpectedly stopping were both increased by 10 percentage points. For ease of illustration, we kept journey time, waiting time, and probability of not being able to board constant.

To apply the model, we need to standardize the ride features. For this process, we first take every value, subtract the corresponding mean, and divide by the corresponding standard deviation. As the goal is to compare the revised schedule to baseline performance, we standardized both schedules using means and standard deviations from the original sample. These standardized values represent how far the ride is from the original-schedule average, expressed in standard deviation units. Thus, if a ride has a crowding score of -1 , it indicates that ride was one standard deviation less crowded than the baseline average.

Predicted satisfaction scores were then derived for the two samples. These values reflect difference from the originalschedule average expressed in standard deviation units. So, a predicted satisfaction score of 0.5 indicates a user who is half a standard deviation above average in satisfaction. Therefore, under the original schedule, the average satisfaction level was 0 (by definition). The average for the revised schedule was 0.96 . Thus, the revised schedule leads to an increase of about 1 standard deviation in average satisfaction.

However, it is also important to estimate the range of plausible averages for each sample. Because the model does not include every factor which affects satisfaction, these means represent the central point of a distribution of plausible values. Values closer to the center are more likely, and those further away are less so. As we know the variance of individual level residuals, we can model this distribution and so build an interval which captures the majority of plausible values (the central 95\%).

This interval is valuable in determining whether the predicted difference between the schedules is meaningful. If the intervals for the two schedules heavily overlap, any average difference is dwarfed by model-external factors. If, conversely, they are separated, then the difference is reliable and likely to consistently emerge (if the ride experiences are constant).

To determine the intervals, we need to estimate how adding a random residual term to each individual will affect the average across individuals. In technical terms, we need to determine the sampling distribution for an average of 1000 residuals. As 
discussed in section 3.3.3. this estimate $(\sigma=0.89)$ was found by applying the model to the unseen test data. By the central limit theorem, we can say that the average of $\mathrm{n}$ residuals will be normally distributed with a standard deviation equal to $\sigma /$ $\sqrt{n} .95 \%$ of values would therefore fall between $1.96 * \sigma / \sqrt{n}$ above and below the predicted mean. ${ }^{3}$

The resulting intervals are shown in Figure 5. The points show the sample averages, whereas the intervals are shown by the lines. As discussed earlier, the revised schedule has a higher average. The lines show the range of plausible values. In this case, the intervals are completely distinct. Thus, the proposed changes lead to a reliable increase in satisfaction.

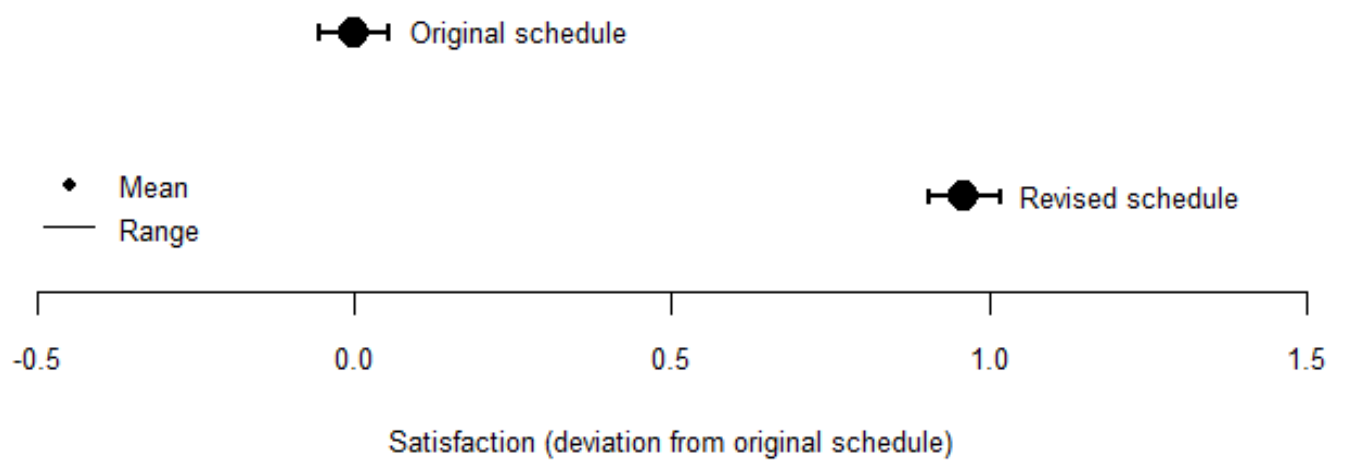

Figure 5. Comparing predicted satisfaction values. Average satisfaction values for 1000 simulated users under two schedules. Point shows average satisfaction. Line shows a range of plausible values (95\% intervals).

\footnotetext{
${ }^{3}$ An alternative approach would be to re-run the prediction a number of times, adding residual error onto each individual observation each time. The average will be slightly different each time, so an interval can be empirically determined. We use the analytic solution as it is quicker.
} 


\section{DISCUSSION}

The current research has provided a validated predictive model for passenger satisfaction, incorporating an empiricallycalibrated degree of uncertainty. Cross-validation and regularization algorithms were used to ensure a generalizable model, and a separate test sample was used to provide a fair estimate of performance on new cases. The resulting model can be used to derive predictions of how system changes will affect passenger satisfaction. In addition, the coeffficients from a fuller regression model also provide practical insight into the features which drive satisfaction.

As we see it, the core strength of the current model is its relevance for practice. It takes as inputs concrete, on-the-ground ride features which can be quantified based on simulated system functioning. It then outputs predicted satisfaction values. This ability to generate satisfaction predictions from ride functioning provides a basis for informing decisions about transit systems. This practical utility sets the current work apart from existing models of transit satisfaction, which provide good insight into satisfaction but are not geared towards generating predictions.

However, as ever, there are a number of ways the current work could be further extended.

First, the model would be of even greater utility to planners if it were connected to broader simulations of transit networks. As reviewed in the introduction, simulations are able to predict the effects of system changes on ride experiences. If our model were incorporated into these simulations, then they could also predict changes on passenger satisfaction. This output would allow more informed operational decisions.

Second, the model could be expanded to include stable individual differences in satisfaction. Some people will tend to give systematically higher satisfaction ratings than others (e.g., Carrel et al., 2016). With ratings of single ride, these dispositional tendencies are simply an additional factor incorporated in the residual term. However, if individuals rate multiple rides, individual baselines can be incorporated into the model. The current study investigated single rides in order to minimize respondent burden and so reach a broad swathe of users. Subsequent studies can focus on tracking smaller groups of respondents to get richer longitudinal information. 
Third, the current work is based on one transit mode in one country. Other factors will be relevant for different transit modes. For example, relative to MRT passengers, pedestrians / cyclists face more dangers from traffic, whereas bus passengers are less likely to have access to real-time arrival information. Furthermore, different populations are likely to have different priorities. These could be representative of different demands on passengers (e.g., importance of punctuality) as well as different experiences (e.g., comfort with crowding). 
Acknowledgement. This work was funded by a Land Transit Innovation Fund grant by the Singapore government to the Institute of High Performance Computing.

\section{References}

Carrel, A., Lau, P. S., Mishalani, R. G., Sengupta, R., \& Walker, J. L. (2015). Quantifying transit travel experiences from the users' perspective with high-resolution smartphone and vehicle location data: Methodologies, validation, and example analyses. Transportation Research Part C: Emerging Technologies, 58, 224-239.

Carrel, A., Mishalani, R. G., Sengupta, R., \& Walker, J. L. (2016). In pursuit of the happy transit rider: dissecting satisfaction using daily surveys and tracking data. Journal of Intelligent Transportation Systems, 20, 345-362.

Carrel, A., Sengupta, R., \& Walker, J. L. (2017). The San Francisco Travel Quality Study: tracking trials and tribulations of a transit taker. Transportation, 44, 643-679.

de Oña, J., de Oña, R., Eboli, L., \& Mazzulla, G. (2013). Perceived service quality in bus transit service: a structural equation approach. Transport Policy, 29, 219-226.

Eboli, L. \& Mazzulla, G. (2007). Service quality attributes affecting customer satisfaction for bus transit. Journal of Public Transportation, 10, 21-34.

Eboli, L. \& Mazzulla, G. (2011) A methodology for evaluating transit service quality based on subjective and objective measures from the passengers point of view. Transport Policy, 18, 172-181.

Ettema, D., Friman, M., Garling, T., Olsson, L. E., \& Fujii, S. (2012). How in-vehicle activities affect work commuters' satisfaction with public transport. Journal of Transport Geography, 24, 215-222.

Ettema, D., Gärling, T., Eriksson, L., Friman, M., Olsson, L. E., \& Fujii, S. (2011). Satisfaction with travel and subjective well-being: Development and test of a measurement tool. Transportation Research Part F: Traffic Psychology and Behaviour, 14, 167-175.

Friedman, J., Hastie, T., Tibshirani, R. (2010). Regularization paths for generalized linear models via coordinate descent. Journal of Statistical Software, 33, 1-22.

Friman, M. (2004). Implementing quality improvements in public transport. Journal of Public transportation, 7, 49-65.

Friman, M., Edvardsson, B., \& Gärling, T. (2001). Frequency of negative critical incidents and satisfaction with public transport services. I. Journal of Retailing and Consumer Services, 8, 95-104. 
Friman, M., \& Fellesson, M. (2009). Service supply and customer satisfaction in public transportation: The quality paradox. Journal of Public transportation, 12, 57-69.

Friman, M., \& Gärling, T. (2001). Frequency of negative critical incidents and satisfaction with public transport services. II. Journal of Retailing and Consumer Services, 8, 105-114.

Friman, M., Fujii, S., Ettema, D., Gärling, T., \& Olsson, L. E. (2013). Psychometric analysis of the satisfaction with travel scale. Transportation Research Part A: Policy and Practice, 48, 132-145.

Joewono, T. B., \& Kubota, H. (2007). User perceptions of private paratransit operation in Indonesia. Journal of Public Transportation, 10, 99-118.

Kuhn, M. (2008). Building predictive models in R using the caret package. Journal of Statistical Software, 28, 1-26.

Lam, W. H., Cheung, C. Y., \& Lam, C. F. (1999). A study of crowding effects at the Hong Kong light rail transit stations. Transportation Research Part A: Policy and Practice, 33, 401-415.

McDonnell, S., \& Zellner, M. (2011). Exploring the effectiveness of bus rapid transit a prototype agent-based model of commuting behavior. Transport Policy, 18, 825-835.

Ory, D. T., \& Mokhtarian, P. L. (2005). When is getting there half the fun? Modeling the liking for travel. Transportation Research Part A: Policy and Practice, 39, 97-123.

Othman, N. B., Legara, E. F., Selvam, V., \& Monterola, C. (2015). A data-driven agent-based model of congestion and scaling dynamics of rapid transit systems. Journal of Computational Science, 10, 338-350.

R Core Team (2015). R: A language and environment for statistical computing [computer software]. R Foundation for Statistical Computing, Vienna, Austria, URL http://www.R-project.org/.

Singapore Land Transit Authority (2017). Public transport utilisation - average daily public transport ridership [Data set; 1995-2016]. Retrieved from https://data.gov.sg/dataset/public-transport-utilisation-average-public-transportridership?resource_id=552b8662-3cbc-48c0-9fbb-abdc07fb377a

Stradling, S. G., Anable, J., \& Carreno, M. (2007). Performance, importance and user disgruntlement: A six-step method for measuring satisfaction with travel modes. Transportation Research Part A: Policy and Practice, 41(1), 98-106.

Stuart, K. R., Mednick, M., \& Bockman, J. (2000).Structural equation model of customer satisfaction for the New York City subway system. Transportation Research Record, 1735,133-137

St-Louis, E., Manaugh, K., van Lierop, D., \& El-Geneidy, A. (2014). The happy commuter: A comparison of commuter satisfaction across modes. Transportation Research Part F: Traffic Psychology and Behaviour, 26, 160-170. 
Tyrinopoulos , Y. \& Aifadopulou, G. (2008). A complete methodology for the quality control of passenger services in the public transport business. European Transport, 38, 1-16

Wahba, M., \& Shalaby, A. (2011). Large-scale application of MILATRAS: case study of the Toronto transit network. Transportation, 38, 889-908. 